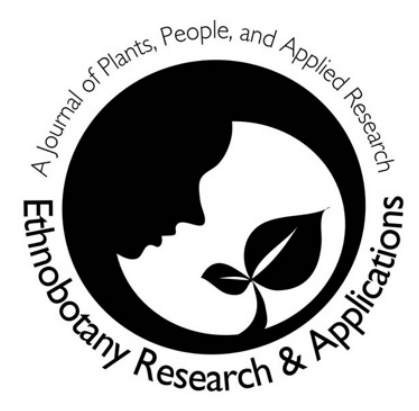

\title{
Perceptions locales des services écosystémiques des aires protégées à l'Est du Burkina Faso
}

\author{
Issaka Ouédraogo, Oumarou Sambaré, Salfo Savadogo, \\ Adjima Thiombiano
}

\section{Research}

\begin{abstract}
Résumé
Contexte: Les écosystèmes offrent d'innombrables services à l'Homme. Cependant, la source pourvoyeuse des services qu'est l'écosystème est mal perçue, entrainant de sérieux problèmes de conservation. Cette étude vise à évaluer les perceptions locales des services écosystémiques et leur source d'approvisionnement.
\end{abstract}

Méthodes: Elle a été conduite dans trois aires protégées et leurs zones environnantes à l'Est du Burkina Faso. Des interviews semi-structurées individuelles ont été réalisées au près de 148 personnes pour recenser les bénéfices qu'elles tirent des aires protégées $(A P)$ et les stratégies locales pour leur pérennisation. Les bénéfices ont été rangés entre les quatre catégories de services écosystémiques (approvisionnement, régulation, culturels et support) et une autre catégorie dénommée " autres services ". Des pourcentages de citations ont été calculés et un test de comparaison statistique a été effectué. Une Analyse en Composantes Principales a été également conduite pour répartir les différents bénéfices par AP.

Résultats: Les populations locales ont énuméré huit zones protégées offrant 39 types de bénéfices, répartis entre les différentes catégories. La catégorie des services d'approvisionnement et celle « autres services " offrent le maximum de services. Les bénéfices offerts varient d'une zone à une autre. Le parc national du $W$ offre le plus de bénéfices $(64,10$ $\%)$. Le statut de conservation de chaque aire protégée influence l'approvisionnement des services. Ainsi, la plupart des services classés par le Millennium Ecosystem Assessment sont fournis par les parcs pendant que ceux de la catégorie « autres services » sont fournis par les zones cynégétiques.
Les populations reconnaissent bien les services écosystémiques avec leurs sources d'approvisionnement et ont proposé 25 solutions pour assurer leur pérennisation.

Conclusion: Les populations locales ont fourni des données importantes pouvant aider à l'élaboration des plans de gestion fiables prenant en compte leurs connaissances et aspirations culturelles.

Mots clés: Conservation, Populations locales, Gestion durable des ressources naturelles, Services écosystémiques, Stratégies locales

\section{Correspondence}

Issaka Ouédraogo*1,3, Oumarou Sambaré ${ }^{1,3}$, Salfo Savadogo 2,3 , Adjima Thiombiano 3

${ }^{1}$ Département des Sciences de la Vie et de la Terre, Institut des Sciences, 01 BP 1757 Ouagadougou 01, Burkina Faso

2Département Substances Naturelles, Institut de Recherche en Sciences Appliquées et Technologies, Centre National de la Recherche Scientifique et Technologique, 03 BP 7047 Ouagadougou 03, Burkina Faso

${ }^{3}$ Laboratoire de Biologie et Ecologie Végétales, Département de Biologie et Physiologie Végétales, Université Joseph KI-ZERBO, 09 BP 848 Ouagadougou 09, Burkina Faso

*Corresponding Author: sonrenoma@yahoo.fr

Ethnobotany Research \& Applications

$20: 13(2020)$ 


\begin{abstract}
Background: Ecosystems provide countless services to humans. However, the source of ecosystem services is poorly perceived by local populations leding to overexploitation. This study aims to assess local perceptions of ecosystem services and their sources of supply.
\end{abstract}

Methods: The study was conducted in three protected areas (PA) and their surrounding zones in eastern Burkina Faso. Open-ended interviews were conducted randomly on 148 persons to identify the benefits people obtain from PA. Local strategies for conservation were also identified. The benefits were classified into the four categories of ecosystem services (provisioning, regulating, cultural and supporting) and another category called "other services". Citation percentages were calculated, and statistical comparison test was carried out. A Principal Component Analysis (PCA) was conducted to break down the different services by PA.

Results: Local people listed eigth protected areas offering 39 types of benefits, divided into the different categories. Provisioning services and category "other services" were the most quoted. The benefits vary from one area to another. The National Park of $W$ offers the most benefits (64.10\%). The conservation status of each PA influences the supply of benefits. Thus, most of the services classified by the Millennium Ecosystem Assessment are provided by the parcs, while those in the "other services" category are provided by the hunting zones. The populations recognize ecosystem services with their sources and proposed 25 solutions to ensure the sustainability of services.

Conclusion: The local people point of view is important for reliable management plans taking into account their cultural knowledge and aspirations.

Keywords: Conservation, Local People, Sustainable use of natural Resources, Ecosystem Services, Local Strategies

\section{Contexte}

Les écosystèmes procurent à l'Homme de nombreux biens et services tels que l'alimentation, l'eau de boisson, le bois de chauffe, les produits médicinaux, la purification de l'air, les bénéfices spirituels, etc. (Costanza et al. 1997, de Groot et al. 2002, Limoges 2009, Fisher et al. 2010, Ouédraogo et al. 2014). Ces biens et services sont regroupés sous le vocable de " services écosystémiques " (Costanza et al. 1997), définis par le MEA (2005) comme « les bénéfices que les populations tirent des écosystèmes ». Le MEA (2005) a regroupé les services écosystémiques en quatre grandes catégories que sont: (i) les services d'approvisionnement ou de production tels que l'alimentation, l'eau, le bois et les fibres; (ii) les services de régulation qui prennent en compte la régulation du climat, des inondations, des maladies, la qualité de l'eau, l'assimilation des déchets; (iii) les services culturels qui prennent en compte les valeurs récréatives, esthétiques, et les bénéfices spirituels; et (iv) les services de support qui concernent la formation des sols, la photosynthèse et les éléments cycliques. Certains de ces services sont parfois négligés et les écosystèmes pourvoyeurs sont mêmes méconnus. Probablement la raison fondamentale qui fait que l'Homme ne prête pas attention aux services offerts par les écosystèmes est qu'il les considère comme un don de la nature (Salzman 2005, Yelkouni 2005). D'autres services sont si discrets que les populations n'arrivent pas à faire le lien entre l'écosystème pourvoyeur et lesdits services. Le cas des services de régulation est très illustratif de cet état de fait. En effet, il n'est pas évident que la population locale sache qu'une bonne pluviométrie ou une épuration des eaux puisse avoir comme source un écosystème forestier. Cette ignorance pose de sérieux problèmes de sécurisation des aires forestières et de leur gestion durable au bénéfice des générations futures. II est donc nécessaire, que les populations soient sensibilisées sur les services et les sources pourvoyeuses pour adopter des comportements favorables à la conservation. La Convention sur la Diversité Biologique (CDB) rappelle à cet effet, l'importance d'intégrer les populations locales dans la gestion de leurs zones d'habitation et celles avoisinantes. Les savoirs locaux dits traditionnels sont reconnus comme indispensables pour une bonne gestion des ressources (Busquet 2006).

Au Burkina Faso, la plupart des travaux sur les savoirs locaux ont concerné la satisfaction des besoins par l'utilisation d'espèces (Taïta 2003, Traoré et al. 2011, Sop et al. 2012), focalisant ainsi la source des services sur l'espèce, et occultant l'écosystème d'où elle est issue. Les perceptions locales sur la source d'approvisionnement qu'est l'écosystème sont alors peu connues. Ces connaissances sont pourtant capitales pour analyser les points de vue des bénéficiaires des services sur les écosystèmes forestiers. De Groot et al. (2002) affirment que les valeurs sociales et les perceptions paysannes sont importantes pour déterminer l'importance des écosystèmes naturels et leurs fonctions pour les sociétés humaines. Les bénéfices des écosystèmes naturels ne sont souvent pas bien compris et plusieurs paysages à fonctions multiples continuent d'être convertis en d'autres types d'utilisation des terres (de Groot 2006). II faudra 
donc pour prendre des décisions objectives relatives à la gestion de la biodiversité, disposer d'informations sur le potentiel des écosystèmes naturels et leur exploitation par l'Homme. Au Burkina Faso, les écosystèmes naturels sont en majorité dominés par des savanes (Fontès \& Guinko 1995), toujours présentes dans les aires protégées. En effet, plusieurs études ont montré le rôle important des aires protégées dans la conservation de la biodiversité (Ouédraogo et al. 2009, Schumann et al. 2010, Gnoumou et al. 2011, Ouédraogo et al. 2011). Ces aires protégées sont des zones de refuge des espèces végétales menacées et rares. Elles présentent une structure assez stable de leurs communautés végétales formant des habitats variés pour les espèces de grands mammifères des savanes tels que l'éléphant, le buffle, le lion, etc. En outre, les droits d'usage traditionnels sont reconnus aux populations riveraines. Ces droits sont entre autres la cueillette des fruits, la récolte des feuilles, des racines et d'écorces pour la pharmacopée, le fauchage de la paille et le ramassage du bois mort pour des usages domestiques. Le caractère naturel des écosystèmes des aires protégées et les droits d'usage traditionnels accordés aux populations riveraines permettent l'obtention de données représentatives sur l'utilisation des ecosystèmes naturels par l'Homme, à des fins de conservation. C'est dans cette optique que le parc national du W (PNW), le parc national d'Arly (PNA), la réserve partielle de faune de Pama nord (RPFPN) ainsi que leurs zones annexes ont été retenus pour cette étude. Leur choix se justifie davantage par le fait qu'elles sont situées à l'Est du Burkina Faso abritant la majorité des aires protégées du pays. Aussi fontelles partie du complexe W-Arly-Pendjari. Ce complexe représente en Afrique de l'Ouest, le plus grand continuum des écosystèmes terrestres (Lamarque 2004). II inclut les trois parties nationales du parc régional du W (Bénin, Burkina Faso, Niger), le parc national de la Pendjari, le parc national d'Arly ainsi que les zones de chasse, les réserves partielles limitrophes et les zones cultivées.

Ce travail a pour objectif global d'analyser les points de vue des populations locales sur les services écosystémiques et leur source à l'Est du Burkina Faso. Plus spécifiquement il s'agit (i) d'appréhender les bénéfices ou avantages que les populations locales tirent des aires protégées à l'Est du Burkina Faso (ii) d'appréhender les services écosystémiques fournis par chaque aire protégée et (iii) d'évaluer les stratégies locales pour la pérennisation des services écosystémiques. Cette étude présume que les perceptions locales dans l'approvisionnement des services écosystémiques varient selon les aires protégées.

\section{Matériel et méthodes \\ Zone d'étude}

La zone d'étude est située dans la région de l'Est du Burkina Faso. Elle recouvre le parc national du $\mathrm{W}$ (PNW), le parc national d'Arly (PNA), la réserve partielle de faune de Pama Nord (RPFPN) et leurs zones environnantes. Elle s'étend entre les latitudes $11^{\circ} 10^{\prime}$ et $12^{\circ} 40^{\prime}$ Nord et les longitudes $0^{\circ} 20^{\prime}$ et $2^{\circ} 30^{\prime}$ Est (Figure 1). Elle est caractérisée par un climat de type soudano-sahélien et soudanien, avec une variante un peu plus aride au Nord et une variante un peu plus humide au Sud (Fontès \& Guinko 1995). La moyenne pluviométrique annuelle est comprise entre $700 \mathrm{~mm}$ et $900 \mathrm{~mm}$. La pluviométrie moyenne annuelle des 33 dernières années est de 809,33 $\mathrm{mm}$. La périphérie des aires protégées est occupée par plusieurs groupes ethniques dont les principaux sont les Gourmantché $(85 \%)$ et les Peuls (12\%) (Mbayngone et al. 2008). Ils vivent principalement d'agriculture et d'élevage. Sur le plan culturel, $80 \%$ de la société Gourmantché est animiste et effectue des sacrifices dans des lieux de culte et/ou des bois sacrés (Thiombiano et al. 2002). Les aires protégées subissent des feux précoces d'aménagement entre novembre et décembre. Les aires protégées sont préservées de l'agriculture et du déboisement. La chasse traditionnelle et le pâturage domestique y sont prohibés alors que dans les champs, le pâturage, les feux incontrôlés et l'exploitation des produits forestiers ligneux et non ligneux de subsistance sont des pratiques courantes (Belem et al. 2007). Cependant, certaines activités illégales sont constatées dans les aires protégées. II s'agit principalement du braconnage et la fraude pour la pêche et la récolte du miel, du pâturage domestique et de l'empiètement des superficies par l'agriculture.

\section{Collecte des données Choix des villages}

Des interviews ont été conduites dans les villages et hameaux de cultures riverains aux aires protégées (AP). Aux environs du PNW ce sont les villages de Barpoua, Gnibwama, Kaabougou, Kondio, Nangwanli, Tapoa djerma et Toptchiagou. Au niveau du PNA les enquêtes ont concerné Djabwanli, Nagaré, Naguèlga, Saborga, Tanli et Tougou ainsi que Boubouanga, Boudiago, Bourahima, Dabilyima, Guematoogo, Marbori, Namaanou, Penkada et Yipéna à proximité de la réserve partielle de faune de Pama Nord (RPFPN). La plupart des villages choisis disposent d'organisations internes que sont les Comités Villageois de Gestion de la Faune (CVGF) et des postes forestiers. Les coordonnées géographiques de chaque village et/ou hameaux de culture ont été enregistrées. Ces coordonnées ont permis de générer la Figure 1 qui illustre la distribution des localités visitées. 

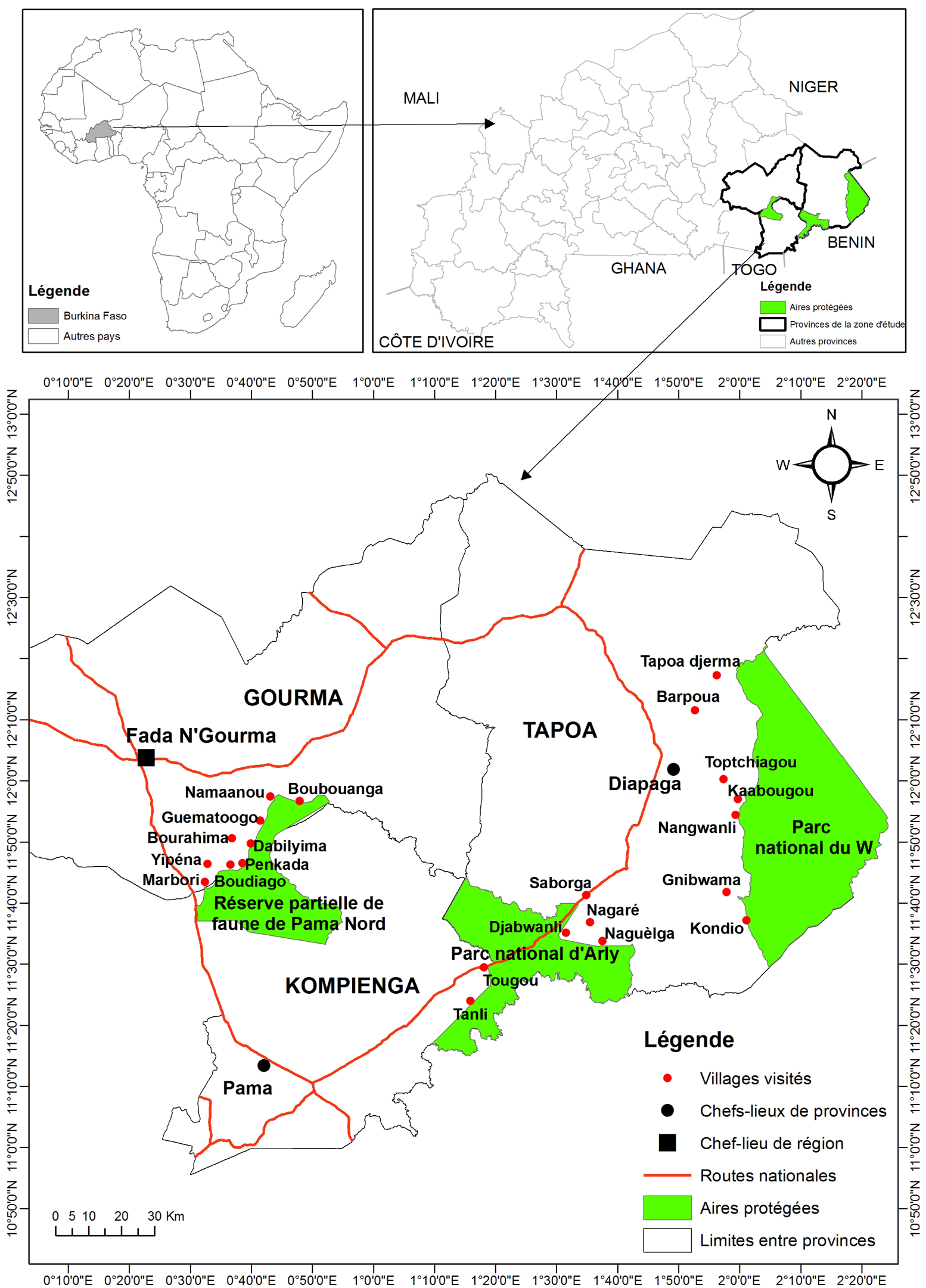

Figure 1. Distribution des localités visitées

Figure 1. Distribution of the visited localities 


\section{Mode d'échantillonnage}

Un échantillonnage aléatoire orienté a été conduit à la fois sur des personnes adultes de sexe masculin et féminin. Le choix des personnes a consisté à leur expliquer les objectifs de l'étude (contribuer à la gestion des ressources naturelles), afin d'obtenir leur consentement à répondre aux questions. Certaines personnes rencontrées, en majorité des femmes, n'étaient pas disposées à prendre part aux entretiens. Cela s'explique par les pesanteurs socioculturelles (Sop et al. 2012) qui obligent les femmes à prendre l'avis de leur époux avant tout propos. Dans certaines localités, les responsables administratifs villageois ont été avisés avant la conduite des interviews. La taille de l'échantillon a été de 51 personnes pour les terroirs environnants du PNW, 47 pour ceux du PNA et 50 pour ceux de la RPFPN, soit au total 148 personnes.

\section{Déroulement des interviews}

Une Interview Semi-Structurée (ISS) individuelle a été conduite pour évaluer l'importance des AP pour les populations riveraines. Elle a consisté d'abord à vérifier si la population reconnaissait l'existence d'aires protégées dans l'entourage des villages/hameaux de cultures. Ensuite les entretiens ont permis de recenser les bénéfices que chaque aire protégée citée offre à la population. Cela prend en compte l'ensemble des profits et avantages procuré par les aires protégées. Les questions ont été centrées par la suite sur les principales aires protégées (AP) que sont le PNW, le PNA et la RPFPN. Ainsi, des stratégies locales pour la pérennisation des services écosystémiques à travers la préservation des aires protégées ont été recensées. Une enquête complémentaire sur la gestion des zones de chasse et des zones villageoises d'intérêts cynégétiques (ZOVIC) a été également conduite. Elle a concerné les responsables des services forestiers qui sont principalement les chefs des postes forestiers et les responsables des CVGF.

\section{Analyse des données}

Les aires protégées citées par les riverains et leurs bénéfices pour la population ont été énumérés. Les bénéfices des aires protégées ont été rangés dans les quatre catégories de services écosystémiques. Certains bénéfices fournis par les AP, ne sont pas issus du fonctionnement des écosystèmes et, ont été classés sous la dénomination " autres services ». En effet, le terme "fonctions des écosystèmes » est la capacité des processus naturels et des composantes à produire des services qui satisfont les besoins de l'Homme (de Groot et al. 2002). Les pourcentages de citations des informations reçues ont été calculés pour évaluer l'importance des services offerts par aire protégée. Les pourcentages de citations ont permis également d'évaluer le consensus des interviewés sur les solutions préconisées pour la pérennisation des bénéfices tirés des AP. Un test non paramétrique de Wilcoxon/Kruskal-Wallis au seuil de $5 \%$ a été effectué pour comparer les différents services offerts par aire protégée ainsi que les différentes solutions préconisées pour leur continuité. Une Analyse en Composantes Principales (ACP) a permis de regrouper les services offerts (variables initiales) selon les aires protégées (individus). L'analyse en composantes principales définit sur la base des variables initiales, des variables synthétiques appelées composantes principales qui vont résumer au mieux l'information contenue dans le tableau de départ. L'analyse en composantes principales établit par la suite une corrélation entre les composantes principales et les variables initiales. La corrélation est bonne, lorsque la valeur du coefficient de corrélation entre une variable initiale et une composante principale est supérieure ou égale 50 $\%$. Chaque composante principale a également une capacité de stockage de l'information résumée. L'efficacité de stockage de l'information d'une composante principale est mesurée par la proportion de sa valeur propre par rapport à la somme de toutes les valeurs propres. Plus elle est élevée, plus l'information stockée est importante (Glèlè Kakaï \& Kokodé 2004). Les différents tests ont été conduits dans le logiciel $\mathrm{R}$ version 3.6.3 (The R Foundation for Statistical Computing 2020).

\section{Résultats}

\section{Aires protégées selon les riverains}

Huit zones ont été citées par les populations riveraines comme protégées des actions anthropiques. Ce sont le parc national du W (PNW), le parc national d'Arly (PNA), la zone villageoise d'intérêt cynégétique (ZOVIC) rattachée au PNW, la ZOVIC rattachée au PNA, la zone de chasse rattachée au PNW, la zone de chasse rattachée au PNA, la réserve partielle de faune de Pama Nord (RPFPN) et la chaîne gréseuse du Gobnangou. Ces huit aires protégées se répartissent en deux groupes que sont les parcs nationaux (PNW et PNA) et les zones cynégétiques. Les zones cynégétiques comprennent les zones de chasse rattachées au PNW et au PNA, la RPFPN qui est une réserve partielle dans laquelle la chasse sportive est pratiquée et les ZOVIC rattachées aux PNW et au PNA. La chaine du Gobnangou est une importante chaine gréseuse de la partie Est du Burkina Faso qui affleure dans les deux parcs nationaux (PNW et PNA). 
Tableau 1: Services écosystémiques et leurs pourcentages de citations dans huit zones protégées de l'Est du Burkina Faso Table 1: Ecosystem services and their percentages of citation in eight protected zones in eastern Burkina Faso

\begin{tabular}{|c|c|c|c|c|c|c|c|c|c|c|}
\hline Bénéfices des aires protégées & $\begin{array}{l}\text { Catégories } \\
\text { de services }\end{array}$ & PNW & $\begin{array}{l}\text { ZOVIC- } \\
\text { W }\end{array}$ & $\begin{array}{c}\text { Zone } \\
\text { chasse-W }\end{array}$ & PNA & $\begin{array}{l}\text { ZOVIC- } \\
\text { Arly }\end{array}$ & $\begin{array}{c}\text { Zone } \\
\text { chasse- } \\
\text { Arly }\end{array}$ & $\begin{array}{l}\text { Chaine du } \\
\text { Gobnangou }\end{array}$ & RPFPN & $\begin{array}{c}\text { Ensemble } \\
\text { des } \\
\text { zones }\end{array}$ \\
\hline Paille & $\mathrm{AP}$ & 62,75 & 23,53 & 15,69 & 51,06 & 23,4 & 14,89 & 14,89 & 48 & 84,46 \\
\hline Plantes médicinales & AP & 41,18 & 25,49 & 7,84 & 12,77 & 12,77 & 4,26 & 4,26 & 22 & 43,92 \\
\hline Bois mort (bois de chauffe) & AP & 17,65 & 15,69 & 11,76 & 25,53 & 14,89 & 4,26 & 8,51 & 0 & 32,43 \\
\hline $\begin{array}{l}\text { Fruits (pain de singe, tamarin, } \\
\text { karité, igname sauvage...) }\end{array}$ & AP & 41,18 & 19,61 & 3,92 & 19,15 & 6,38 & 0 & 0 & 6 & 32,43 \\
\hline Bois pour la construction & AP & 15,69 & 9,8 & 13,73 & 21,28 & 8,51 & 0 & 0 & 4 & 24,32 \\
\hline $\begin{array}{l}\text { Viande sauvage issue de la } \\
\text { chasse sportive }\end{array}$ & AP & 0 & 5,88 & 17,65 & 0 & 6,38 & 34,04 & 0 & 8 & 23,65 \\
\hline $\begin{array}{l}\text { Poissons issus de la pêche par } \\
\text { les riverains }\end{array}$ & AP & 0 & 0 & 0 & 48,94 & 2,13 & 4,26 & 0 & 0 & 17,57 \\
\hline $\begin{array}{l}\text { Pâturage d'animaux domestiques } \\
\text { (frauduleux) }\end{array}$ & AP & 7,84 & 11,76 & 5,88 & 0 & 4,26 & 0 & 6,38 & 0 & 12,16 \\
\hline $\begin{array}{l}\text { Viande sauvage (animaux } \\
\text { chassés par les riverains) }\end{array}$ & AP & 0 & 17,65 & 1,96 & 0 & 10,64 & 0 & 2,13 & 0 & 10,14 \\
\hline Eau de boisson & AP & 5,88 & 1,96 & 0 & 4,26 & 0 & 0 & 2,13 & 6 & 6,76 \\
\hline Fibres & AP & 1,96 & 0 & 0 & 6,38 & 4,26 & 0 & 2,13 & 0 & 4,73 \\
\hline Miel (frauduleux) & AP & 3,92 & 1,96 & 0 & 2,13 & 2,13 & 2,13 & 0 & 0 & 4,05 \\
\hline $\begin{array}{l}\text { Matériaux de construction (sable, } \\
\text { graviers...) }\end{array}$ & AP & 0 & 0 & 0 & 2,13 & 0 & 0 & 6,38 & 0 & 2,7 \\
\hline Bois pour l'artisanat & AP & 5,88 & 0 & 0 & 0 & 0 & 0 & 0 & 0 & 2,03 \\
\hline Semences d'espèces végétales & AP & 0 & 0 & 0 & 2,13 & 0 & 0 & 0 & 0 & 0,68 \\
\hline Vision/connaissance de la faune & CT & 19,61 & 5,88 & 0 & 8,51 & 0 & 0 & 0 & 16 & 16,89 \\
\hline Sacrifices & CT & 3,92 & 0 & 0 & 14,89 & 2,13 & 2,13 & 2,13 & 0 & 8,11 \\
\hline Tourisme & CT & 3,92 & 0 & 0 & 2,13 & 0 & 0 & 0 & 0 & 2,03 \\
\hline Augmentation de la pluviométrie & RG & 15,69 & 0 & 0 & 12,77 & 0 & 2,13 & 2,13 & 22 & 18,24 \\
\hline Lutte contre les vents & RG & 3,92 & 0 & 0 & 2,13 & 0 & 2,13 & 2,13 & 2 & 4,05 \\
\hline Air frais & RG & 1,96 & 0 & 0 & 0 & 0 & 0 & 0 & 4 & 2,03 \\
\hline Purification de l'air & RG & 0 & 0 & 0 & 0 & 0 & 0 & 0 & 4 & 1,35 \\
\hline
\end{tabular}




\begin{tabular}{|c|c|c|c|c|c|c|c|c|c|c|}
\hline Bénéfices des aires protégées & $\begin{array}{l}\text { Catégories } \\
\text { de services }\end{array}$ & PNW & $\begin{array}{c}\text { ZOVIC- } \\
\text { W }\end{array}$ & $\begin{array}{c}\text { Zone } \\
\text { chasse-W }\end{array}$ & PNA & $\begin{array}{l}\text { ZOVIC- } \\
\text { Arly }\end{array}$ & $\begin{array}{c}\text { Zone } \\
\text { chasse- } \\
\text { Arly }\end{array}$ & $\begin{array}{l}\text { Chaine du } \\
\text { Gobnangou }\end{array}$ & RPFPN & $\begin{array}{c}\text { Ensemble } \\
\text { des } \\
\text { zones }\end{array}$ \\
\hline Lutte contre les maladies & RG & 0 & 0 & 0 & 0 & 0 & 0 & 0 & 2 & 0,68 \\
\hline Refuge d'espèces végétales & SU & 17,65 & 1,96 & 0 & 0 & 0 & 0 & 0 & 4 & 8,11 \\
\hline Fertilisation des sols & SU & 0 & 0 & 0 & 2,13 & 0 & 0 & 0 & 0 & 0,68 \\
\hline Refuge d'espèces animales & SU & 1,96 & 0 & 0 & 0 & 0 & 0 & 0 & 0 & 0,68 \\
\hline $\begin{array}{l}\text { Génération de revenus pour la } \\
\text { caisse du village }\end{array}$ & AS & 1,96 & 25,49 & 13,73 & 0 & 38,3 & 12,77 & 0 & 8 & 33,11 \\
\hline $\begin{array}{l}\text { Emplois temporaires pour les } \\
\text { jeunes (ouverture des pistes) }\end{array}$ & AS & 17,65 & 3,92 & 0 & 17,02 & 0 & 0 & 0 & 0 & 12,84 \\
\hline $\begin{array}{l}\text { Emplois permanents pour les } \\
\text { jeunes (recrutement de pisteurs) }\end{array}$ & AS & 1,96 & 0 & 1,96 & 6,38 & 0 & 8,51 & 0 & 4 & 7,43 \\
\hline $\begin{array}{l}\text { Obtention de forages grâce aux } \\
\text { ZOVIC }\end{array}$ & AS & 0 & 0 & 0 & 6,38 & 10,64 & 0 & 0 & 0 & 5,41 \\
\hline $\begin{array}{l}\text { Génération de revenus pour la } \\
\text { population }\end{array}$ & AS & 0 & 7,84 & 3,92 & 0 & 0 & 0 & 0 & 0 & 4,05 \\
\hline $\begin{array}{l}\text { Obtention d'écoles grâce aux } \\
\text { ZOVIC }\end{array}$ & AS & 0 & 1,96 & 0 & 4,26 & 6,38 & 0 & 0 & 0 & 4,05 \\
\hline Obtention de vivres & AS & 0 & 0 & 5,88 & 0 & 0 & 0 & 0 & 0 & 2,03 \\
\hline $\begin{array}{l}\text { Contacts avec des } \\
\text { correspondants }\end{array}$ & AS & 0 & 0 & 0 & 0 & 2,13 & 2,13 & 0 & 0 & 1,35 \\
\hline Prévention des conflits & AS & 1,96 & 0 & 0 & 2,13 & 0 & 0 & 0 & 0 & 1,35 \\
\hline Formations (voyage d'étude) & AS & 1,96 & 0 & 0 & 0 & 2,13 & 0 & 0 & 0 & 1,35 \\
\hline $\begin{array}{l}\text { Connaissance de hautes } \\
\text { personnalités }\end{array}$ & AS & 1,96 & 0 & 0 & 0 & 0 & 0 & 0 & 0 & 0,68 \\
\hline Création de CVGF & AS & 1,96 & 0 & 0 & 0 & 0 & 0 & 0 & 0 & 0,68 \\
\hline \multicolumn{2}{|c|}{ Pourcentage de service fourni par aire protégée } & 64,10 & 41,03 & 30,77 & 56,41 & 46,15 & 33,33 & 28,21 & 38,46 & 100 \\
\hline
\end{tabular}

NB: AP: Approvisionnement; RG: Régulation; SU: Support; CT: Culturels; AS: Autres-services; CVGF: Comité Villageois de Gestion de la Faune; PNW: Parc National du W, PNA: Parc National d'Arly, RPFPN: Réserve Partielle de Faune de Pama Nord, ZOVIC: Zone Villageoise d'Intérêt Cynégétique 


\section{Bénéfices des aires protégées}

Les populations riveraines ont cité 39 bénéfices offerts par les huit aires protégées. Ces bénéfices se répartissent en deux grands groupes. Les bénéfices issus du fonctionnement des processus écosytémiques couramment appelés services écosystémiques au nombre de 26 , et 13 autres bénéfices dénommés "autres services » qui sont générés indirectement par la présence des aires protégées (Tableau 1). Les 13 " autres services » représentent 33,33\% de l'ensemble des bénéfices. Parmi les 26 services écosystémiques, la catégorie des services la plus diversifiée est celle des services d'approvisionnement $(38,46 \%)$. Elle est suivie par celle des services de régulation (12,82\%). Les deux catégories de services, support et culturels sont les moins diversifiées avec $7,69 \%$ chacune.

Le nombre de bénéfices offert varie aussi selon la zone. Les zones qui offrent le plus de bénéfices sont respectivement le PNW avec $64,10 \%$ de l'ensemble et le PNA avec $56,41 \%$. La chaine du Gobnangou offre le pourcentage le plus faible avec $28,21 \%$ de l'ensemble des bénéfices (Tableau 1). La Figure 2 renseigne davantage sur la contribution de chaque zone par catégorie de services écosystémiques.

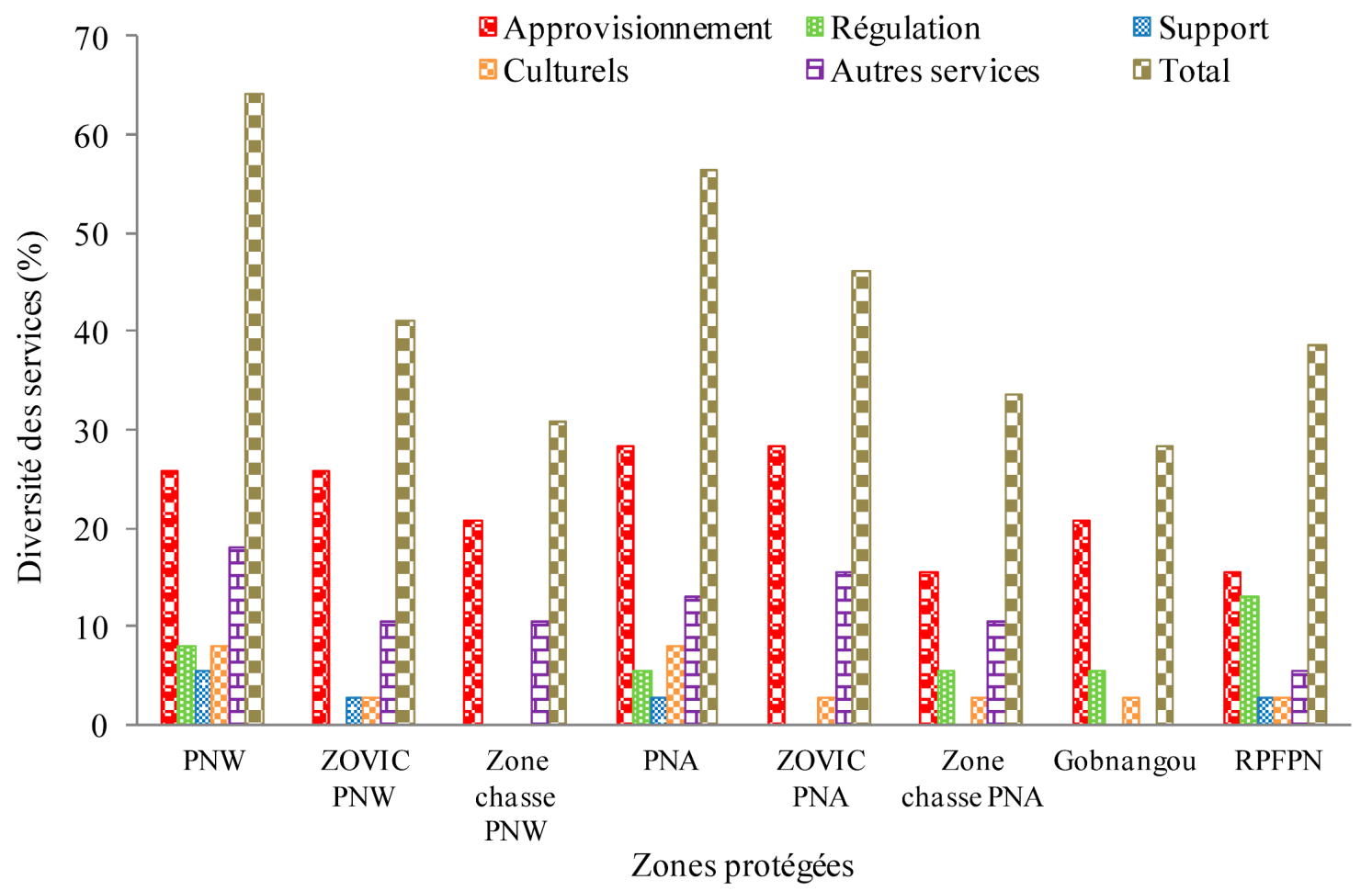

Figure 2. Pourcentage des services écosystémiques par aire protégée

Figure 2. Percentage of ecosystem services per protected area

\section{Importance des bénéfices par aire protégée}

Sur l'ensemble des huit aires protégées citées par les riverains, le service "Paille » est le plus cité par la population riveraine $(84,46 \%)$. Les autres, cités à un pourcentage supérieur ou égal à $20 \%$ sont par ordre d'importance, « Plantes médicinales $(43,92$ $\%)$; Génération de revenus pour la caisse du village $(33,11 \%)$; Bois mort (bois de chauffe) $(32,43 \%)$; Fruits (32,43\%); Bois pour la construction $(24,32 \%)$ et Viande sauvage issue de la chasse sportive $(23,65 \%) »($ Tableau 1). Ces pourcentages de citations diffèrent selon les zones. Le test non paramétrique de Kruskal-Wallis au seuil de $5 \%$ a montré une différence significative entre les bénéfices offerts par les aires protégées ( $p=$ 0,01189).

\section{Répartition des bénéfices par aire protégée}

L'analyse en composantes principales permet de répartir les différents bénéfices selon leur source d'approvisionnement vu que le test de comparaison non paramétrique de Kruskal-Wallis est significatif. L'analyse en composante principale a regroupé les 39 bénéfices qui représentent les variables initiales en sept variables synthétiques ou composantes principales. La première composante (axe 1) a une valeur propre de 14,11 . Elle explique $36,17 \%$ de la répartition des 39 variables initiales en fonction des zones protégées. La deuxième composante principale, avec une valeur propre de 7,87 explique une proportion de $20,19 \%$ des mêmes informations. Le pourcentage cumulé de ces deux axes est de $56,36 \%$ (Tableau 2). La corrélation entre ces deux axes et les 39 variables initiales est donnée dans le Tableau 3. 
Tableau 2. Valeurs propres et pourcentages de variances des composantes principales

Table 2. Eigenvalues and percentages of variances of the main components

\begin{tabular}{llll}
\hline $\begin{array}{l}\text { Composantes } \\
\text { principales }\end{array}$ & $\begin{array}{l}\text { Valeurs } \\
\text { propres }\end{array}$ & $\begin{array}{l}\text { Pourcentage des } \\
\text { variances }\end{array}$ & $\begin{array}{l}\text { Pourcentages cumulés } \\
\text { des variances }\end{array}$ \\
\hline 1 & 14,11 & 36,17 & 36,17 \\
2 & 7,87 & 20,19 & 56,36 \\
3 & 6,23 & 15,98 & 72,35 \\
4 & 4,03 & 10,32 & 82,67 \\
5 & 3,25 & 8,34 & 91,01 \\
6 & 2,19 & 5,62 & 96,63 \\
7 & 1,31 & 3,37 & 100 \\
\hline
\end{tabular}

Le Tableau 3 montre que les variables telles que « Augmentation de la pluviométrie, Bois pour l'artisanat, Bois pour la construction, Connaissance de hautes personnalités, Création de CVGF, Eau de boisson, Emplois temporaires pour les jeunes (ouverture des pistes), Plantes médicinales, Fruits (pain de singe, tamarin, karité, igname sauvage), Lutte contre les vents, Miel (frauduleux), Paille, Prévention des conflits, Refuge d'espèces animales, Refuge d'espèces végétales, Tourisme, Vision/connaissance de la faune » sont positivement corrélées à l'axe 1 (pourcentage de corrélation $\geq 60$ $\%)$. Ces variables s'opposent principalement à celles comme "Génération de revenus pour la caisse du village, Viande sauvage issue de la chasse sportive » et à un faible niveau à d'autres telles que " Cadeaux venant des touristes, Contacts avec des correspondants, Génération de revenus pour la population, Obtention de vivres, Viande sauvage (animaux chassés par les riverains) ॥.

Quant à l'axe 2, il est positivement corrélé aux variables telles que "Bois mort (bois de chauffe), Fertilisation des sols, Fibres, Poissons issus de la pêche par les riverains, Sacrifices, Semences d'espèces végétales, Obtention de forages grâce aux ZOVIC, Obtention d'écoles grâce aux ZOVIC » (pourcentage de corrélation $\geq 60 \%$ ). Ces variables s'opposent principalement aux services comme « Air frais, Lutte contre les maladies, Purification de l'air, Pâturage d'animaux domestiques ».

L'essentiel de ces informations est résumé dans le graphique de corrélation des variables formé par les deux axes 1 et 2 (Figure 3). Quatre grandes entités peuvent être ainsi discriminées conformément à la Figure 4 qui représente aussi la distribution des individus (aires protégées) en quatre grandes entités. Sur la Figure 4, le PNW est situé du côté positif de l'axe 1 et le PNA du côté positif de l'axe 2. Ceci montre que les bénéfices positivement corrélés à l'axe 1 sont cités pour le compte du PNW tandis que le PNA offre les bénéfices positivement corrélés à l'axe 2. La RPFPN située du côté négatif de l'axe 2 forme le troisième groupe et offre les bénéfices qui sont situés du coté négatif de l'axe 2.

Les ZOVIC rattachées au PNW et au PNA, les zones de chasse rattachées au PNW et au PNA et la chaîne du Gobnangou situées du coté négatif de l'axe 1 forment le quatrième groupe. Ce groupe peut être réparti en deux sous-groupes (la ZOVIC rattachée au PNA d'un côté et les trois autres d'un autre côté). Cette subdivision montre que même si ce quatrième groupe offre les mêmes bénéfices, ils peuvent à l'intérieur, être scindés en deux. C'est le cas de la ZOVIC rattachée au PNA qui fournit en plus deux bénéfices que sont «Obtention de forages grâce aux ZOVIC, Obtention d'écoles grâce aux ZOVIC » pendant que les ZOVIC rattachées au PNW et au PNA, les zones de chasse rattachées au PNW et au PNA et la chaîne du Gobnangou offrent majoritairement les bénéfices qui s'opposent à l'axe 1.

\section{Bénéfices issus des parcs nationaux}

Au regard du graphe de corrélation des variables (Figure 3), 25 bénéfices sont offerts à la fois par les PNW et PNA. De ces 25, 19 sont classés dans les quatre catégories de services écosystémiques. Ces 19 services proviennent du fonctionnement et des processus écosystémiques. Ils sont généralement liés aux différents droits d'usage reconnus aux riverains tels que les services d'approvisionnement (paille, bois morts, fibres, fruits, eau de boisson, espèces pour la pharmacopée, sacrifices).

\section{Bénéfices issus des zones cynégétiques}

Toujours, selon la Figure 3, les bénéfices « Contacts avec des correspondants, Cadeaux venant des touristes, Génération de revenus pour la caisse du village, Génération de revenus pour la population, Obtention de vivres, Viande sauvage issue de la chasse sportive, Viande sauvage (animaux chassés par les riverains) » sont fournis par l'ensemble des zones de chasse et les ZOVIC. II y a également les bénéfices « Obtention de forages grâce aux ZOVIC et Obtention d'écoles grâce aux ZOVIC » qui sont fournis par la ZOVIC rattachée au PNA. Ainsi, les zones de chasse et les ZOVIC pourvoient majoritairement les bénéfices classés « autres services ". Les interviews avec les différents responsables de CVGF et des postes forestiers ont permis d'étayer cela. II ressort de ces interviews que les CVGF bénéficient des retombées liés à la gestion et à la conservation des zones protégées. 
Tableau 3. Corrélation entre variables initiales et composantes principales Table 3- Correlation between initial variables and main components

\begin{tabular}{|c|c|c|c|}
\hline Variables initiales & Codes des variables & Axe 1 & $\overline{\text { Axe } 2}$ \\
\hline Air frais & Ame_vie & 0,41 & $-0,64$ \\
\hline Augmentation de la pluviométrie & Aug_pluv & 0,70 & $-0,25$ \\
\hline Bois mort (bois de chauffe) & Boi_mort & 0,51 & 0,71 \\
\hline Bois pour l'artisanat & Boi_arts & 0,83 & $-0,35$ \\
\hline Bois pour la construction & Boi_cons & 0,62 & 0,49 \\
\hline Cadeaux venant des touristes & Kdo_tour & $-0,42$ & 0,09 \\
\hline Connaissance de hautes personnalités & Conn_vip & 0,83 & $-0,35$ \\
\hline Contacts avec des correspondants & Correspt & $-0,43$ & 0,18 \\
\hline Création de CVGF & Cre_cvgf & 0,83 & $-0,35$ \\
\hline Eau de boisson & Eau_bois & 0,81 & $-0,25$ \\
\hline Emplois permanents pour les jeunes (recrutement de pisteurs) & Rec_pist & 0,08 & 0,23 \\
\hline Emplois temporaires pour les jeunes (ouverture des pistes) & Ouv_pist & 0,92 & 0,34 \\
\hline Plantes médicinales & Esp_phar & 0,78 & $-0,41$ \\
\hline Fertilisation des sols & Fer_sols & 0,37 & 0,82 \\
\hline Fibres & Rec_fibr & 0,38 & 0,83 \\
\hline Formations (voyage d'étude) & Formatio & 0,42 & $-0,04$ \\
\hline Fruits (pain de singe, tamarin, karité, igname sauvage...) & Rec_pfnl & 0,90 & $-0,05$ \\
\hline Génération de revenus pour la caisse du village & Gen_cais & $-0,51$ & 0,06 \\
\hline Génération de revenus pour la population & Gen_popu & $-0,29$ & $-0,12$ \\
\hline Lutte contre les maladies & Lut_mala & 0,02 & $-0,50$ \\
\hline Lutte contre les vents & Lut_vent & 0,73 & $-0,20$ \\
\hline Matériaux de construction (sable, graviers...) & Mat_cons & $-0,09$ & 0,23 \\
\hline Miel (frauduleux) & Miel & 0,64 & 0,18 \\
\hline Obtention de forages grâce aux ZOVIC & Obt_forr & $-0,01$ & 0,70 \\
\hline Obtention de vivres & Obt_vivr & $-0,30$ & $-0,07$ \\
\hline Obtention d'écoles grâce aux ZOVIC & Obt_ecol & $-0,03$ & 0,73 \\
\hline Paille & Paille & 0,93 & $-0,04$ \\
\hline Pâturage d'animaux domestiques (frauduleux) & Pat_andm & 0,05 & $-0,27$ \\
\hline Poissons issus de la pêche par les riverains & Peche & 0,34 & 0,84 \\
\hline Prévention des conflits & Evi_conf & 0,90 & 0,40 \\
\hline Purification de l'air & Bon_air & 0,02 & $-0,50$ \\
\hline Refuge d'espèces animales & Ref_anim & 0,83 & $-0,35$ \\
\hline Refuge d'espèces végétales & Ref_vegt & 0,83 & $-0,48$ \\
\hline Sacrifices & Lie_sacr & 0,51 & 0,81 \\
\hline Semences d'espèces végétales & Rec_seme & 0,37 & 0,82 \\
\hline Tourisme & Act_tour & 0,96 & 0,09 \\
\hline Viande sauvage (animaux chassés par les riverains) & Chs_rive & $-0,34$ & 0,06 \\
\hline Viande sauvage issue de la chasse sportive & Via_sauv & $-0,56$ & $-0,16$ \\
\hline Vision/connaissance de la faune & Vis_rive & 0,85 & $-0,38$ \\
\hline
\end{tabular}

NB: CVGF: Comité Villageois de Gestion de la Faune, ZOVIC: Zone Villageoise d'Intérêt Cynégétique 


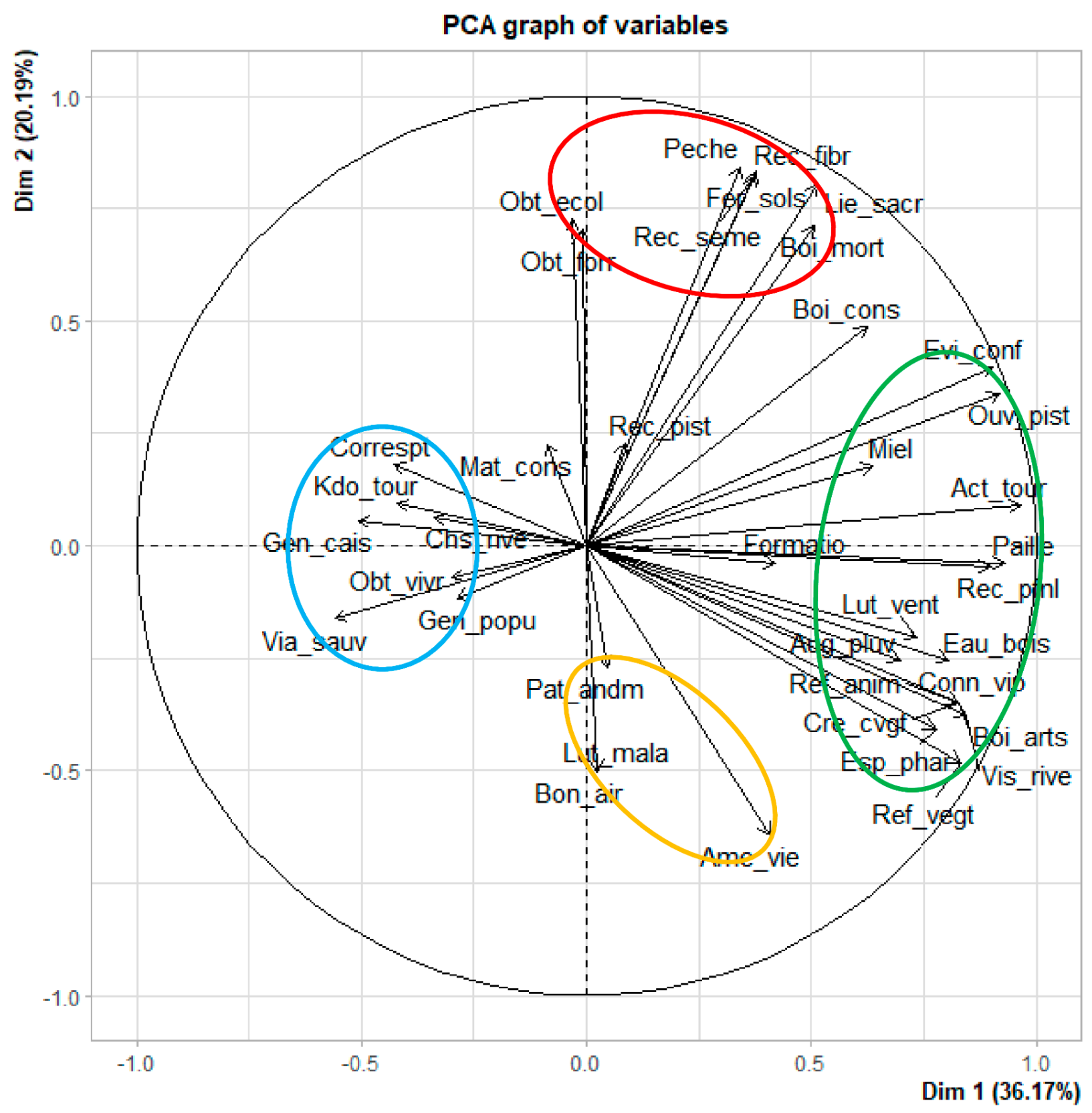

Figure 3. Distribution des services dans le plan factoriel de l'ACP

NB: Les abréviations correspondent aux codes des variables du tableau 3.

Figure 3: Distribution of services in the PCA factor plan

Note: Abbreviations correspond to the variable codes in table 3.

Au niveau des zones de chasse et selon le cahier des charges, après l'abattage d'un animal, la venaison est répartie selon la clé suivante: deuxquart à la population locale, un-quart au concessionnaire et un-quart au service forestier. La part de la population locale est remise de façon tournante aux CVGF des villages riverains à la zone de chasse. Les CVGF décident librement de l'utilisation de leur viande. Dans certains villages la viande est partagée entre la population, dans d'autres, elle est vendue sous forme de brochettes ou en tas au bénéfice de la population. Chaque habitant peut s'en acquérir et consommer de la viande sauvage selon ses moyens. 


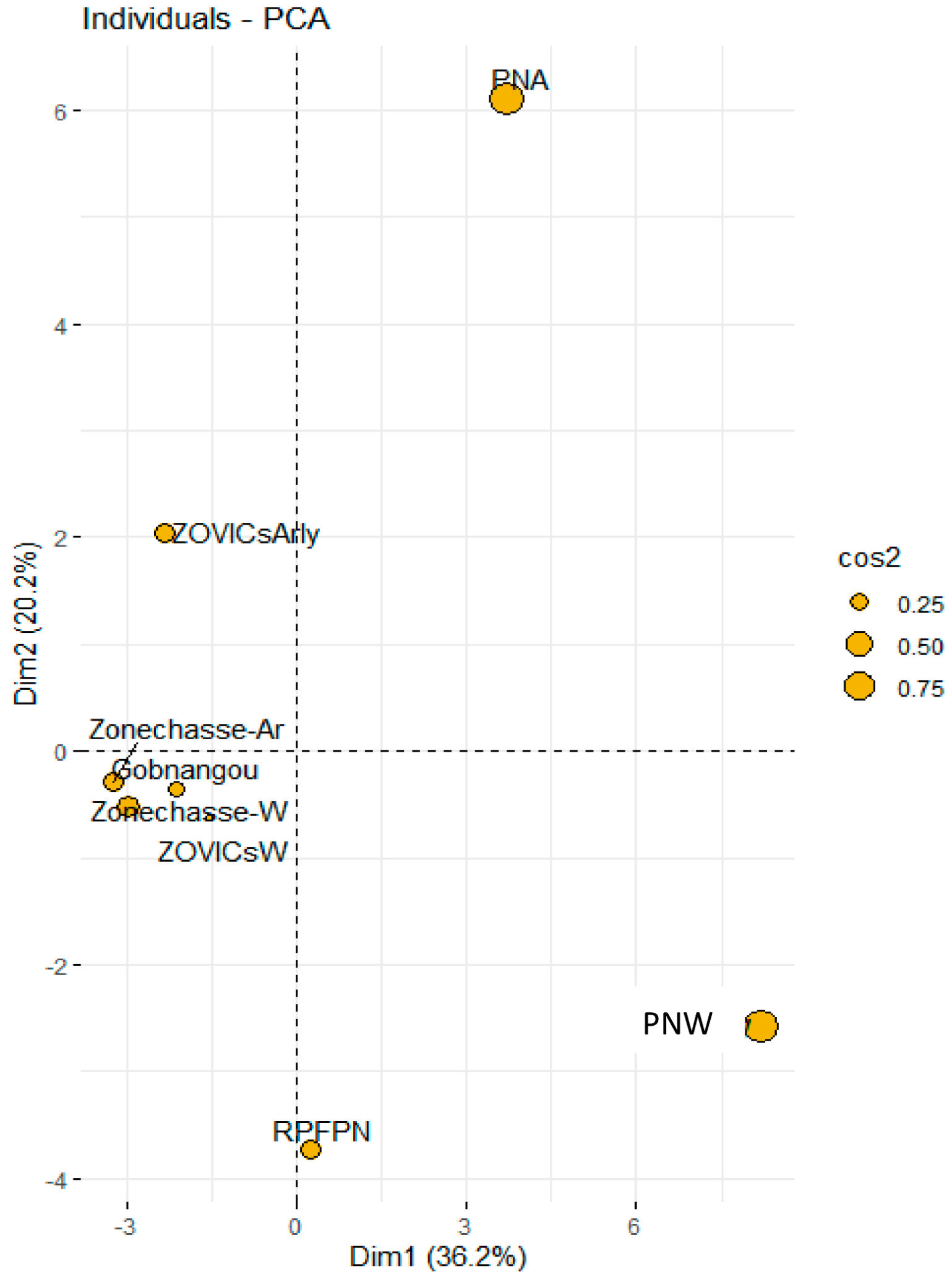

Figure 4. Distribution des aires protégées dans le plan factoriel de l'ACP Figure. Distribution of protected areas in the PCA factor plan 
Le revenu de cette vente est reversé dans les caisses du village pour le financement des activités de développement du même village, telles que la construction ou la réfection d'écoles, de centres de santé et de promotion sociale, de forages. L'argent peut également être octroyé aux habitants sous forme de petits crédits pour permettre à certains de démarrer des activités génératrices de revenus ou de prêts à certaines personnes démunis pour résoudre un problème ponctuel (soins de santé, achat de vivres).

Les concessionnaires des zones de chasse offrent également des vivres à la population riveraine pour nouer une bonne cohésion. Ils viennent parfois aussi en appui au village à travers des dons de médicaments aux postes de santé ou à l'installation de forages. En outre, lorsque les chasseurs partent pour le safari, ils distribuent souvent des cadeaux aux populations des villages qu'ils traversent. De retour, certains qui ont atteint leurs objectifs, sont très heureux et offrent des cadeaux aux pisteurs qui les ont accompagnés. D'autres chasseurs les prennent entièrement en charge pendant plusieurs années pendant que d'autres parrainent des élèves en leur octroyant des bourses ou des fournitures scolaires.

Au niveau des ZOVIC, où la petite chasse est pratiquée, les villages riverains s'organisent en CVGF afin de mieux gérer les retombées. Chaque chasseur de petit safari paie des permis journaliers (4000 F à 7500 F CFA) aux CVGF. L'argent des permis est reversé dans le compte des CVGF à Diapaga (chef de la province de la Tapoa) pour les ZOVIC rattachées au PNW et au PNA et à Fada N'Gourma (chef de lieu de la province du Gourma) pour la ZOVIC rattachée à la RPFPN. Cet argent est ensuite partagé de façon égale entre les CVGF des villages riverains à une même ZOVIC à la fin de la saison de chasse. En outre, la viande issue de la petite chasse est distribuée entre trois entités selon une clé de répartition bien établie: quatre-dixième aux CVGF, trois-dixième au concessionnaire, et trois-dixième au poste forestier. La part des riverains est remise aux CVGF de la ZOVIC de façon tournante. La gestion de la viande est à l'initiative du CVGF. Ainsi, elle est soit vendue, soit partagée. En cas de vente, l'argent est reversé directement dans une caisse installée dans le village. Les fonds vont être utilisés pour financer des activités de développement à l'intérieur du village. L'école du village de Toptchiagou (village riverain au PNW), aux dires de la population a été entièrement financée par la caisse du village, alimentée par la vente de la viande issue de la petite chasse. De cette manière, les ZOVIC génèrent des revenus aux populations locales.

\section{Proposition de solutions pour la pérennisation des services}

Diverses solutions, au nombre de 25 , ont été proposées par les riverains des trois principales aires protégées, soit pour améliorer la conservation des AP, soit pour éviter la perte des bénéfices évoqués plus haut. Les solutions les plus consensuelles, citées d'au moins $10 \%$ sur l'ensemble de la zone d'étude consistent à lutter contre les feux de brousse; à lutter contre la coupe du bois vert; à sensibiliser les populations que l'AP est un bien public et sur les méthodes de coupe; à lutter contre le braconnage et à renforcer la surveillance. Les proportions de citations des solutions varient d'une aire protégée à l'autre (Tableau 4), tandis que le test non paramétrique de comparaison de moyenne de Wilcoxon/Kruskal Wallis au seuil de $5 \%$ a montré une différence non significative entre les solutions proposées ( $p=$ 0,4774).

\section{Discussion}

\section{Services des aires protégées}

Les populations ont cité une grande diversité de bénéfices générés par les AP. Parmi ces bénéfices certains ont été classés dans les quatre catégories définies par le MEA (2005) et d'autres ont été nommés " autres services ». Cette classification vient de la définition même de services écosytémiques. En effet selon le MEA (2005) les services écosystémiques sont des bénéfices que les populations tirent des écosystémiques. Pour de Groot et al. (2002), ces servies sont en fait des retombées des fonctions des écosytèmes. Puisqu'une AP est un écosystème, c'est à dire « une communauté complexe et dynamique de plantes, d'animaux, de microorganismes et d'éléments abiotiques (MEA 2005, Kumar \& Kumar 2007) », elle est constituée d'éléments biotiques et abiotiques qui interagissent. Ces interactions génèrent des services qui satisfont les besoins de l'Homme. Burkhard et al. (2011) ont défini alors les services écosystémiques comme une contribution des structures et fonctions des écosystèmes (en combinaison à d'autres inputs) au bien-être de l'Homme. Cependant la catégorie classée « autres services " n'est pas directement générée par ce fonctionnement des AP. Ce sont des retombées de la gestion des $\mathrm{AP}$ et indirectement liés à leur présence.

De l'ensemble des bénéfices cités, les services d'approvisionnement sont plus importants. Selon la dénomination du MEA (2005), ces services regroupent les services tels que la nourriture, l'eau, les fibres, les produits pharmaceutiques. Ces services servent directement à la satisfaction des besoins de l'Homme. Selon la vision 
anthropocentrique du concept des services écosystémiques (Fisher et al. 2010, Burkhard et al. 2011), la satisfaction des besoins de l'Homme est primordiale. C'est dans cet ordre d'idées que les services d'approvisionnement ont été plus cités par les populations, mettant ainsi en avant-plan les besoins de l'Homme. C'est ce qui justifie le point de vue de Burkhard et al. (2011) qui soutient que la demande en services écosystémiques est élevée dans les zones à forte présence humaine.
Ouédraogo et al. (2014) ayant recensé les espèces utilisées dans les quatre catégories de services écosystémiques trouvent plus d'espèces (84,42\% du total des espèces recensées) dans la catégorie des services d'approvisionnement que les trois autres catégories de services. Cissé et al. (2018) ont obtenu des résultats similaires dans le bassin versant de Boura dans la zone soudanienne du Burkina Faso.

Tableau 4. Solutions de conservation des aires protégées proposées par les populations riveraines Table 4. Local people solutions for protected areas conservation

\begin{tabular}{|c|c|c|c|c|}
\hline Propositions des solutions de conservation & PNW & PNA & RPFPN & Total \\
\hline Lutter contre les feux de brousses & 39,22 & 25,53 & 19,61 & 28,38 \\
\hline Lutter contre la coupe du bois vert & 37,25 & 27,66 & 17,65 & 27,70 \\
\hline $\begin{array}{l}\text { Sensibiliser les populations sur le fait que les AP sont des biens } \\
\text { publics }\end{array}$ & 17,65 & 6,38 & 7,84 & 10,81 \\
\hline Renforcer la surveillance & 11,76 & 14,89 & 5,88 & 10,81 \\
\hline Lutter contre le braconnage & 9,80 & 12,77 & 7,84 & 10,14 \\
\hline Empêcher les gens d'accéder aux parcs & 21,57 & 0,00 & 3,92 & 8,78 \\
\hline Augmenter le salaire du personnel & 9,80 & 0,00 & 0,00 & 3,38 \\
\hline Augmenter le personnel de surveillance & 5,88 & 2,13 & 1,96 & 3,38 \\
\hline Lutter contre le pâturage frauduleux dans les AP & 1,96 & 4,26 & 3,92 & 3,38 \\
\hline $\begin{array}{l}\text { Augmenter la distance entre les villages riverains et les AP en } \\
\text { reculant les limites des parcs au cours de nouveaux tracés }\end{array}$ & 1,96 & 0,00 & 5,88 & 2,70 \\
\hline Impliquer les riverains dans la gestion & 5,88 & 2,13 & 0,00 & 2,70 \\
\hline Améliorer les conditions de travail des gestionnaires des AP & 3,92 & 0,00 & 1,96 & 2,03 \\
\hline Interdire la création de nouveaux champs & 0,00 & 0,00 & 5,88 & 2,03 \\
\hline Intensifier l'agriculture & 1,96 & 2,13 & 0,00 & 1,35 \\
\hline $\begin{array}{l}\text { Dédommager les personnes qui subissent les pertes liées à la faune } \\
\text { (éléphants, hyènes, lions) }\end{array}$ & 0,00 & 4,26 & 0,00 & 1,35 \\
\hline $\begin{array}{l}\text { Restaurer les zones dégradées des AP avec des espèces locales } \\
\text { adaptées }\end{array}$ & 1,96 & 0,00 & 1,96 & 1,35 \\
\hline $\begin{array}{l}\text { Aménager les terres dégradées à vocation agricole au profit des } \\
\text { populations riveraines }\end{array}$ & 1,96 & 0,00 & 0,00 & 0,68 \\
\hline Construire des points d'eau et des salines pour la faune dans les AP & 1,96 & 0,00 & 0,00 & 0,68 \\
\hline Accorder des dotations de vivres aux populations riveraines & 1,96 & 0,00 & 0,00 & 0,68 \\
\hline Interdire la cueillette des fruits immatures & 0,00 & 2,13 & 0,00 & 0,68 \\
\hline $\begin{array}{l}\text { Partager les retombées liées aux AP jusqu'à la base (population } \\
\text { riveraine) }\end{array}$ & 0,00 & 2,13 & 0,00 & 0,68 \\
\hline $\begin{array}{l}\text { Inciter les populations riveraines à planter des espèces exotiques } \\
\text { pour les utiliser en construction et dans la culture de l'igname et du } \\
\text { manioc }\end{array}$ & 0,00 & 2,13 & 0,00 & 0,68 \\
\hline $\begin{array}{l}\text { Rechercher des partenaires pour soutenir les actions de conservation } \\
\text { des AP }\end{array}$ & 1,96 & 0,00 & 0,00 & 0,68 \\
\hline Recruter des pisteurs autochtones et non étrangers & 0,00 & 0,00 & 1,96 & 0,68 \\
\hline Traiter et résoudre efficacement les conflits fonciers & 0,00 & 2,13 & 0,00 & 0,68 \\
\hline
\end{tabular}

NB: AP: Aire protégée, PNW: Parc National du W, PNA: Parc National d'Arly, RPFPN: Réserve Partielle de Faune de Pama Nord, ZOVIC: Zone Villageoise d'Intérêt Cynégétique

Les aires protégées de la région de l'Est étant des écosystèmes forestiers, offrent un important lot d'autres services à l'image de tout écosystème forestier. Elles sont des zones de refuge pour de multiples espèces animales et végétales. Plusieurs études phytosociologiques ont mentionné ce service refuge des AP (Schumann et al. 2010, Ouédraogo et al. 2011). Mbayngone et al. (2008) précisent que 
certaines espèces telles que Pseudocedrela kotschyi (Schweinf.) Harms, Prosopis africana (Guill. \& Perr.) Taub., Burkea africana Hook., Securidaca longipedunculata Fresen., Pericopsis laxiflora (Benth.) Meeuwen, Stereospermum kunthianum Cham., Bombax costatum Pellegr. \& Vuill. qui sont considérées disparues ou menacées en zones hors protégées sont bien représentées dans la réserve partielle de faune Pama. En outre la plupart des travaux ayant conduit à la découverte de nouvelles espèces pour le Burkina Faso ont été menées à l'intérieur des $A P$, dénotant ainsi le caractère refuge des AP. Plusieurs études ont également montré le rôle important des écosystèmes forestiers dans l'atténuation des gaz à effet de serre. Selon Djomo (2015), deux virgule six milliards de tonnes de carbones sont stockés par les écosystèmes forestiers terrestres sur huit milliards de tonnes produits chaque année par les activités anthropiques à travers la combustion des énergies fossiles et le changement d'utilisation des terres. Même si des données relatives aux stocks de carbone des AP du Burkina Faso ne sont pas disponibles à ce jour, elles contribuent significativement à l'atténuation des gaz à effet de serre à travers la séquestration de carbone. C'est ce que les populations riveraines ont mentionné à travers les services purification de l'air et amélioration de la vie. Limoges (2009) ayant travaillé sur les bandes riveraines mentionne que la purification de l'air est l'une des fonctions les plus importantes de cet écosystème. Les bandes riveraines contribuent à la lutte contre la pollution diffuse corroborant ainsi le point de vue des populations riveraines.

Les aires protégées constituent des habitats appropriés pour plusieurs espèces emblématiques telles que le lion, l'éléphant, le buffle, le guépard... Ainsi plusieurs structures (équipes de football, partis politiques...) s'identifient à ces espèces animales faisant référence à leur force ou à leur rapidité. Ces services, même s'ils n'ont pas été rapportés par les populations locales, relèvent de la catégorie des services culturels offerts par les AP et la biodiversité en générale. En outre plusieurs études ont montré l'importance socio-économique des AP (Taïta 2003, Dabouineau \& Ponsero 2009). Guo et al. (2001) précisaient que pour amener les gens à comprendre l'importance des services éosystémiques il faut les convertir en valeur économique.

Les bénéfices classés " autres services » sont également abondants. Certains participent à l'entretien des pistes pour accroître le niveau du tourisme. Les AP offrent encore d'autres types d'emplois à la population. UICN/PACO (2009) rapporte que les aires protégées emploient du personnel des villages riverains comme: pisteurs, surveillants, cuisiniers, serveurs, guides, etc. Certains avantages comme la construction d'écoles et l'octroi de vivres sont reçus des partenaires. Ces résultats corroborent ceux de Mbayngone et Thiombiano (2011) qui ont montré que la réserve partielle de faune de Pama est pourvoyeuse de plusieurs avantages tels que la construction d'écoles et de dépôts pharmaceutiques.

\section{Importance des services selon le statut des aires protégées}

La diversité des services varie d'une aire protégée à l'autre. Le statut des aires protégées en matière de conservation de la biodiversité serait un atout pour qu'une AP offre une gamme variée de services. En effet, selon le statut, les actions de conservation en faveur de la biodiversité sont plus contraignantes favorisant ainsi un épanouissement de la biodiversité pour plus d'approvisionnement en services écosystémiques. En rappel, le PNW a un statut de parc national et, depuis novembre 2002, United Nations Educational, Scientific and Cultural Organization (UNESCO) lui a conféré le statut de "réserve transfrontalière de la biosphère", la toute première en Afrique de l'Ouest (UICN/PACO 2009). Le PNA bénéficiait du titre de "parc national » sans statut juridique officiel depuis 1978 (UICN/PACO 2009). C'est tout récemment en 2015 que le PNA a été juridiquement constitué (Décret $N^{\circ}$ 2015- 916 /PRES-TRANS promulguant la loi $\mathrm{n}^{\circ}$ 042-2015/CNT du 02 juillet 2015). La réserve partielle de faune de Pama a été créée par l'Arrêté $n^{\circ} 6070 / S E F$ du $03 / 08 / 1955$ et garde à nos jours le statut de réserve partielle de faune (UICN/PACO 2009). Sur la base de la classification de I'Union Internationale pour la Conservation de la Nature (UICN), le PNW et le PNA sont rattachés à la catégorie II des AP et la réserve partielle de faune de Pama à la catégorie IV. La catégorie II concerne les parcs nationaux, gérés principalement dans le but de protéger les écosystèmes et à des fins récréatives. La catégorie IV concerne les aires de gestion des habitats ou des espèces à des fins de conservation avec une possibilité de prélèvement (Héritier 2007). Ces différents statuts ont favorisé un accroissement différent de la biodiversité dans les trois localités. Les études phytosociologiques conduites dans ces localités montrent que la richesse spécifique du PNW est de 622 espèces végétales contre 490 espèces végétales au PNA et 450 espèces végétales à la RPFPN (Mbayngone et al. 2008, Ouédraogo et al. 2011, Nacoulma 2012). En outre, certains travaux ont trouvé une relation évidente entre la biodiversité et l'approvisionnement des services écosystémiques (Sandifer et al. 2015). Selon Ouédraogo et al. (2014), la richesse spécifique d'un écosystème contribue à augmenter sa capacité à pourvoir des services écosystémiques. 
En somme, la littérature écologique montre que la diversité fonctionnelle soutenue par la biodiversité est un facteur clé dans la fourniture des services écosystémiques de façon durable (Sandifer et al. 2015).

Néanmoins, la plupart des bénéfices classés dans « autres services " est plus rattachée aux zones cynégétiques. Par contre les quatre catégories de services écosystémiques du MEA (2005) sont plus fournies par les parcs nationaux. Le rôle joué par chacune des zones protégées dans la gestion et la conservation des ressources peut expliquer cette répartition. En effet, la ZOVIC est une variante d'aire protégée dont la gestion revient aux autorités villageoises à travers un comité villageois de gestion de la faune (CVGF). Cette gestion concerne l'usage du site pour la petite chasse (Kaboré 2010). Les zones de chasse représentent des parties concédées à des particuliers pour l'organisation de la grande chasse, pendant que les PNW et PNA participent à la conservation en assurant certains droits d'usage aux populations riveraines. L'ensemble des actions de répartition des retombées des AP à travers les ZOVIC et les zones de chasse explique pourquoi la catégorie " autres services " est offert par les ZOVIC et les zones de chasse.

\section{Pérennisation des services écosystémiques à travers la conservation des aires protégées}

Les populations riveraines ont reconnu l'importance des aires protégées à travers les bénéfices qu'elles ont cités. La reconnaissance des zones protégées est un signe d'acceptation du statut de conservation de chaque AP facilitant ainsi le respect des mesures restrictives édictées par le statut des AP. Dans ce sens, les riverains préconisent à un pourcentage d'au moins $10 \%$ de mieux lutter contre les feux de brousse, la coupe abusive du bois, le braconnage et de renforcer la surveillance ainsi que la sensibilisation des populations. Citer telle ou telle zone comme protégée, sous entend que la population accepte de respecter le statut accordé à l'AP. En effet, la méconnaissance de l'existence des AP aux environs des villages est un refus de reconnaitre le statut des AP qui contribuent à la préservation de l'environnement.

La reconnaissance des zones protégées montre aussi la compréhension locale sur le lien existant entre les services écosystémiques et leurs sources que sont les AP. C'est en ce sens que Daily et al. (1997) mentionnaient que peu de personnes sont conscientes du rôle joué par les écosystèmes naturels dans l'approvisionnement des services. Elles ignorent les bénéfices qu'elles tirent des AP, car les jugeant trop élémentaires. L'ignorance de la source des biens et services continue malheureusement de croître surtout dans les zones urbaines (Daily et al. 1997, Salzman 2005). Pourtant, la pérennisation d'une fourniture en services écosystémiques traduit une bonne santé à long terme des écosystèmes pourvoyeurs (Sandifer et al. 2015). Ce principe est bien compris par les populations riveraines lorsqu'elles ont proposé les 25 solutions pour la pérennisation des services écosystémiques. II est également important de prendre en compte les aspirations culturelles des populations locales. Selon Busquet (2006), les valeurs culturelles et spirituelles des espaces et des ressources ne peuvent pas être séparées ni des stratégies de planification ni du mode de vie de la population locale. Dans cette optique, il serait avantageux de mettre l'accent sur la sensibilisation des autorités coutumières et religieuses des villages afin qu'elles soient des relais pour sensibiliser les populations locales. La population écouterait les propos venant d'un chef ou d'un sage du village que n'importe quel agent du service forestier. En effet, dans les localités dominées par des populations à culture chasseur, il faut que le chef lui-même, dépositaire de la tradition, interpelle la population à abandonner le braconnage pour qu'elle ne se sente pas en marge de sa culture. Il faudrait aussi rassurer cette population en sauvant leur tradition. II faut également, pour assurer l'intégrité des limites des AP, amener les populations locales vers une modernisation de l'agriculture afin d'augmenter les rendements et éviter leur empiètement. Cela doit se faire à travers l'octroi de moyens agricoles, mais aussi par des formations et des suivis réguliers et rigoureux.

\section{Conclusion}

Les aires protégées sont connues des populations riveraines. Elles y tirent une gamme variée de bénéfices. Les populations locales reconnaissent chaque aire protégée et les différentes catégories de bénéfices qu'elle pourvoit. Ainsi, les sources d'approvisionnement de chaque service écosystémique sont bien connues des populations riveraines. Les populations locales sont conscientes du risque de disparition des services si les aires protégées ne sont pas préservées. Les différentes solutions préconisées pour la pérennisation des services témoignent de cet état de fait. La diversité et l'importance des services offerts sont fonction du statut de conservation de chaque aire protégée. L'hypothèse de départ est alors confirmée. Les populations connaissent également les activités qui sont autorisées dans chaque catégorie d'aire protégée. Elles ont alors accepté la restriction des activités anthropiques dans les AP édictées par les statuts de conservation. De ce qui précède, les aires protégées constituent sans doute une source immense de services écosystémiques qu'il faut à 
tout prix préserver pour le bien-être de l'Homme. II faudrait alors que les gestionnaires travaillent à prendre en compte les aspirations culturelles des populations pour établir un plan de gestion qui recueillera l'assentiment de tous.

\section{Déclarations}

Liste des abréviations: ACP: Analyse en Composantes Principales; AP: Aire protégée; CVGF: Comité Villageois de Gestion de la Faune; ISS: Interview Semi-structurée; PACO: Programme Afrique Centrale et Occidentale; PNA: Parc National d'Arly; PNW: Parc National du W; RPFPN: Réserve Partielle de Faune de Pama Nord; UICN: Union Internationale pour la Conservation de la Nature; UNESCO: United Nations Educational, Scientific and Cultural Organization; ZOVIC: Zone Villageoise d'Intérêt Cynégétique

Approbation éthique et consentement à la participation: Sans objet

Disponibilité des données et du matériel: Dans le cadre du présent travail, les données traitées sont disponibles pour d'éventuelles demandes par la revue.

Financement: Le programme Loewe Research Centre Biodiversity and Climate (BiK-F) a assuré l'appui financier et logistique qui a facilité les travaux. Conflit d'intérêts: Les auteurs déclarent qu'il n'y a aucun conflit d'intérêts.

Contributions des auteurs: 10 a élaboré le protocole de recherche, collecté les données et a rédigé ce présent article, OS et SS ont aidé à l'analyse des données et à la fourniture de littératures pour consolider la discussion, AT a défini les objectifs et les grandes lignes pour la réalisation du travail.

\section{Remerciements}

Nous sommes reconnaissants au programme Loewe Research Centre Biodiversity and Climate (BiK-F) pour son soutien financier et logistique, qui a facilité le travail sur le terrain. Nous sommes également reconnaissants aux populations locales vivant autour du parc national du W, du parc national d'Arly et de la réserve partielle de faune de Pama Nord pour avoir partagé leurs connaissances. En outre, nous sommes reconnaissants aux reviewers anonymes pour leurs commentaires constructifs

\section{Littérature citée}

Belem B, Nacoulma BMI, Gbangou R, Kambou S, Hansen HH, Gausset Q, Lund S, Raebild A, Lompo D, Ouédraogo M, Theilade I, Boussim JI. 2007. Use of Non-Wood Forest Products by local people bordering the "Parc National Kaboré Tambi", Burkina Faso. The Journal of Transdisciplinary Environmental Studies 6 (1):1-21.
Burkhard B, Kroll F, Nedkov S, Müller F. 2011. Mapping ecosystem service supply, demand and budgets. Ecological Indicators doi:10.1016/j.ecolind.2011.06.019.

Busquet MB. 2006. Des stratégies intégrées durables: savoir écologique traditionnel et gestion des espaces et des ressources. Vertigo 7(2):1-8.

Cissé M, Bationo BA, Traoré S, Boussim IJ. 2018. Perception d'espèces agroforestières et de leurs services écosystémiques par trois groupes ethniques du bassin versant de Boura, zone soudanienne du Burkina Faso. Bois et Forêts des Tropiques 338:29-42. Doi: https://doi. org/10.19182/bft2018.338.a31680.

Costanza R, Darge R, de Groot R, Farber S, Grasso M, Hannon B, Limburg K, Naeem S, Oneill R, Paruelo J, Raskin GR, Sutton P, Belt VDM. 1997. The value of the world's ecosystem services and natural capital. Nature 387:253-260. doi:10.1038/387253a0.

Dabouineau L, Ponsero A. 2009. Comment évaluer les services rendus par les écosystèmes ? Ou combien d'Euros vaut une abeille ou un hectare de zone humide?. Le Râle d'Eau 137:9-17.

Daily GC, Alexander S, Ehrlich PR, Goulder L, Lubchenco J, Matson PA, Mooney HA, Postel S, Schneider SH, Tilman D, George M, Woodwell GM. 1997. Ecosystem services: Benefits Supplied to Human Societies by Natural Ecosystems. Issues in Ecology 1(2):1-18.

De Groot R, Wilson MA, Boumans RMJ. 2002. A typology for the classification, description and valuation of ecosystem functions, goods and services. Ecological Economics 41:393-408. doi:10.1016/S0921-8009(02)00089-7.

De Groot R. 2006. Function-analysis and valuation as a tool to assess land use conflicts in planning for sustainable, multifunctional landscapes. Landscape and Urban Planning 75:175-186. doi:10.1016/j.landurbplan.2005.02.016.

Djomo A. 2015. Climate change mitigation, forest ecosystems: measurement and modelling of biomass and carbon. First edition. IFED Publishing. Kingston, Canada.

Fisher B, Polasky S, Sterner T. 2010. Conservation and human welfare: economic analysis of ecosystem services. Environ Res Econ. doi:10.1007/s10640010-9415-0.

Fontès J, Guinko S. 1995. Carte de la végétation et de l'occupation du sol du Burkina Faso. Notice explicative. Ministère de la Coopération française, Projet Campus (88 313 101), Toulouse, France.

Glèlè Kakaï R, Kokodé GG. 2004. Techniques statistique univariées et multivariées: applications sur ordinateur. Cotonou, Bénin. 
Gnoumou A, Bognounou F, Hahn K, Thiombiano A. 2011. Woody plant diversity and stand structure in the Comoe-Leraba Reserve Southwestern Burkina Faso (West Africa). Journal of Biological Sciences 1:1-13.

Guo Z, Xiao X, Gan Y, Zheng Y. 2001. Ecosystem functions, services and their values - a case study in Xingshan County of China. Ecological Economics 38:141-154.

Héritier S. 2007. Les parcs nationaux, entre conservation durable et développement local. Géocarrefour 82(4): 171-175

Kaboré A. 2010. Brousse des uns, aire protégée des autres, histoire du peuplement, perceptions de la nature et politique des aires protégées dans le Gourma burkinabè: l'exemple de la réserve partielle de faune de Pama. Thèse, The graduate Institute, Genève, Suisse.

Kumar M, Kumar P. 2007. Valuation of the ecosystem services: A psycho-cultural perspective. Ecological Economics doi:10.1016/j.ecolecon.2007.05.008.

Lamarque F. 2004. Les grands mammifères du complexe WAP. CIRAD, Montpellier, France.

Limoges B. 2009. Biodiversité, services écologiques et bien-être humain. Le naturaliste Canadien, 133:15-19.

Mbayngone E, Thiombiano A, Hahn-Hadjali K, Guinko S. 2008. Caractéristiques écologiques de la végétation ligneuse du sud-est du Burkina Faso (Afrique de l'Ouest): le cas de la réserve de Pama. Candollea 63:7-33.

Mbayngone E, Thiombiano A. 2011. Dégradation des aires protégées par l'exploitation des ressources végétales: cas de la réserve partielle de faune de Pama, Burkina Faso (Afrique de l'Ouest). Fruits 66:187-202. doi:10.1051/fruits/2011027.

MEA (Millennium Ecosystem Assessment), 2005. Ecosystems and human well-being: synthesis. Washington, DC: Island Press.

Nacoulma MIB. 2012. Dynamique et strategies de conservation de la végétation et de la phytodiversité du complexe écologique de Parc National du W du Burkina Faso. Thèse Unique, Université de Ouagadougou, Burkina Faso.

Ouédraogo I, Nacoulma BMI, Hahn K, Thiombiano A. 2014. Assessing ecosystem services based on indigenous knowledge in south-eastern Burkina Faso (West Africa). International Journal of Biodiversity Science, Ecosystem Services \& Management doi: 10.1080/21513732.2014.950980.

Ouédraogo O, Schmidt M, Thiombiano A, Hahn K, Guinko S, Zizka G. 2011. Magnoliophyta, Arly National Park, Tapoa, Burkina Faso. Check List 7 (1):85-100.
Ouédraogo O, Thiombiano A, Hahn-Hadjali K, Guinko S. 2009. Diversité et dynamique de la végétation ligneuse juvénile du Parc National d'Arly (Burkina Faso). Candollea 64(2):257-278.

Salzman J. 2005. Creating markets for ecosystem services: notes from the field, New York University Law Review 80:600:101-184.

Sandifera PA, Sutton-Grierb AE, Ward BP. 2015. Exploring connections among nature, biodiversity, ecosystem services, and human health and wellbeing: Opportunities to enhance health and biodiversity conservation. Ecosystem Services 12:1152.

Schumann K, Wittig R, Thiombiano A, Becker U, Hahn K. 2010. Impact of land-use type and bark-and leaf-harvesting on population structure and fruit production of the baobab tree (Adansonia digitata L.) in a semi-arid savanna, West Africa. Forest Ecology and Management 260 (11):2035-2044.

Sop TK, Oldeland J, Bognounou F, Schmiedel U, Thiombiano A. 2012. Ethnobotanical knowledge and valuation of woody plants species: a comparative analysis of three ethnic groups from the sub-Sahel of Burkina Faso. Environment, Development and Sustainability 14 (5): 627-649.

Taïta P. 2003. Use of woody plants by locals in Mare aux Hippopotames Biosphere Reserve in western Burkina Faso. Biodiversity Conservation 12:12051217. doi:10.1023/A:1023045316329.

Thiombiano A, Ouôba P, Guinko S. 2002. Place des Combretaceae dans la société gourmantché à l'est du Burkina Faso. Etudes Flor. Vég. Burkina Faso 7:17-22.

Traoré L, Ouédraogo I, Ouédraogo A, Thiombiano A. 2011. Perceptions, usages et vulnérabilité des ressources végétales ligneuses dans le Sud-Ouest du Burkina Faso. International Journal of Biological and Chemical Sciences 5:258-278.

UICN/PACO, 2009. Evaluation de l'efficacité de la gestion des aires protégées: aires protégées du Burkina Faso. Jamana Services. Ouagadougou. Burkina Faso.

Yelkouni M. 2004. Gestion d'une ressource naturelle et action collective: le cas de la forêt de Tiogo au Burkina Faso. Thèse Unique de Doctorat. Université d'Auvergne-Clermont I. 